\title{
Genetic Differences in Barley Govern the Responsiveness to N-Acyl Homoserine Lactone
}

\author{
Abhishek Shrestha, ${ }^{1}$ Ahmed Elhady, ${ }^{1}$ Shimaa Adss, ${ }^{1}$ Gwendolin Wehner, ${ }^{2}$ Christoph Böttcher, ${ }^{3}$ \\ Holger Heuer, ${ }^{1}$ Frank Ordon, ${ }^{2}$ and Adam Schikora ${ }^{1, \dagger}$ \\ ${ }^{1}$ Julius Kühn-Institut (JKI), Federal Research Centre for Cultivated Plants, Institute for Epidemiology and Pathogen \\ Diagnostics, Messeweg 11/12, 38104 Braunschweig, Germany \\ 2 Julius Kühn-Institut (JKI), Federal Research Centre for Cultivated Plants, Institute for Resistance Research and \\ Stress Tolerance, Erwin-Baur-Str. 27, 06484 Quedlinburg, Germany \\ ${ }^{3}$ Julius Kühn-Institut (JKI), Federal Research Centre for Cultivated Plants, Institute for Ecological Chemistry, Plant \\ Analysis and Stored Product Protection, Königin-Luise-Straße 19, 14195 Berlin, Germany
}

Accepted for publication 29 May 2019.

\begin{abstract}
Enhanced resistance in barley (Hordeum vulgare) against pathogens, such as the powdery mildew-causing fungus Blumeria graminis f. sp. hordei, is of high importance. The beneficial effects of bacterial quorum sensing molecules on resistance and plant growth have been shown in different plant species. Here, we present the effects of the $\mathrm{N}$-3-oxotetradecanoyl-L-homoserine lactone (oxo-C14-HSL) on the resistance of different barley genotypes. Genetically diverse accessions of barley were identified and exposed to the beneficial, oxo-C14-HSL-producing bacterium Ensifer meliloti or the pure $\mathrm{N}$-acyl homoserine lactone $(\mathrm{AHL})$ molecule. Metabolic profiling along with expression analysis of selected genes and physiological assays revealed that the capacity to react varies among different barley genotypes. We
\end{abstract}

ABSTRACT

demonstrate that upon pretreatment with $\mathrm{AHL}$ molecule, $A H L-$ primable barley genotype expresses enhanced resistance against $B$. graminis f. sp. hordei. We further show that pretreatment with AHL correlates with stronger activation of barley MAP kinases and regulation of defense-related $P R 1$ and $P R 17 b$ genes after a subsequent treatment with chitin. Noticeable was the stronger accumulation of lignin. Our results suggest that appropriate genetic background is required for AHL-induced priming. At the same time, they bear potential to use these genetic features for new breeding and plant protection approaches.

Keywords: agriculture, barley, crop, genetic diversity, microbiome, molecular biology, $\mathrm{N}$-acyl homoserine lactone, priming

\section{Corresponding author: A. Schikora; adam.schikora@julius-kuehn.de}

Funding: Support was provided by the German Research Foundation (Deutsche Forschungsgemeinschaft, DFG), grant SCHI 1132/11, which supported the work of A. Shrestha, and the Federal Ministry of Education and Research (BMBF) for supporting the work of $\mathrm{G}$. Wehner and A. Schikora managed by the Projektträger Jülich (PtJ) (grant FKZ 031B0196B and C).

Author contributions: Ab.S., H.H., F.O., and A.S. conceived the research project. Ab.S. performed pathogen assay, gene expression analysis, assessment of MAP kinase activity and assessment of phenolic compounds and analyzed the data. A.E. performed pathogen assay, gene expression analysis and assessment of phenolic compounds, S.A. performed the assessment of phenolic compounds, G.W. performed the PCA analysis and selection of genotypes and C.B. performed the metabolite profiling. Ab.S. and A.S. wrote the manuscript

*The $\boldsymbol{e}$-Xtra logo stands for "electronic extra" and indicates that five supplementary figures and two supplementary tables are published online.

The author(s) declare no conflict of interest.

(C) 2019 The American Phytopathological Society
Priming for enhanced resistance has long tradition in agriculture, this phenomenon called "sensitization" was used since the 1930s. Upon priming, plants respond stronger and faster to a pathogen attack, which result in robust resistance and secure higher yield. Today, various molecules that induce priming are known and include the following: low concentrations of salicylic acid (SA), benzothiadiazole (BTH), or $\beta$-aminobutyric acid (BABA) (Conrath et al. 2002; Mauch-Mani et al. 2017). In addition, the local and systemic accumulation of azelaic acid occurring during systemic acquired resistance (SAR), primed Arabidopsis thaliana for enhanced SA production and therefore resulted in enhanced resistance to Pseudomonas syringae (Jung et al. 2009). Many recent reports suggested that bacterial quorum sensing (QS) molecules might induce priming in plants. One of the best-studied group of QS molecules is the group of $N$-acyl homoserine lactones (AHL) produced by numerous Gram-negative bacteria to monitor the density of populations. Communication between bacterial individuals, based on the concentration of AHL, or other QS molecules (Fuqua and Winans 1994; Kaplan and Greenberg 1985), is 
today a well-accepted phenomenon. In plants, AHL application was shown to change gene expression, as well as alter the composition of the proteome and root development (Bai et al. 2011; Mathesius et al. 2003; Ortiz-Castro et al. 2008; Schenk et al. 2012; Schikora et al. 2011; von Rad et al. 2008). A study demonstrating the impact of AHL-producing Serratia liquefaciens strain on tomato (Solanum lycopersicum) provided the first evidence that AHL influence plant immunity (Schuhegger et al. 2006); plants inoculated with S. liquefaciens strain MG1, which produces $\mathrm{C} 4-$ and C6homoserine lactones during root colonization (Gantner et al. 2006), were protected against Alternaria alternata. In contrast, plants inoculated with the AHL-negative S. liquefaciens mutant MG44 displayed susceptible phenotype (Schuhegger et al. 2006). Similar results were observed after inoculation with Serratia plymuthica strain HRO-C48 when compared with its AHL-negative spl $\Gamma$ mutant. The HRO-C48 strain protected cucumber plants (Cucumis sativus) against Pythium aphanidermatum, as well as tomato and bean (Phaseolus vulgaris) plants from infection with Botrytis cinerea (Pang et al. 2009). Moreover, our own results show that in A. thaliana, resistance to $P$. syringae was enhanced after inoculation with Ensifer meliloti (Sinorhizobium meliloti) strain

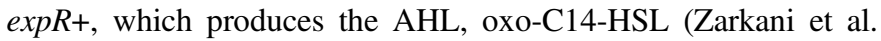
2013). In addition, commercial AHL were shown to enhance resistance against biotrophic and hemibiotrophic bacterial and fungal pathogens (Schikora et al. 2016).

Plant response to AHL appears to be specific to the particular AHL molecule. Analysis of proteins accumulated in response to either oxo-C12-HSL or oxo-C16:1-HSL revealed substantial differences (Mathesius et al. 2003). Consistently, an application of C6-HSL, oxo-C10-HSL, or oxo-C14-HSL resulted in specific transcriptional responses (Schenk et al. 2014). Several reports assessed the molecular basis of priming. Treatment with benzothiadiazole enhanced the accumulation of inactive form of mitogen-activated protein kinases (MAPKs), those could be activated upon following challenge (Beckers et al. 2009). Histone methylation and acetylation in the promoter regions of the defense-associated transcription factors was postulated to participate in priming (Jaskiewicz et al. 2011). Whether the expression or activity of MAPKs and chromatin modification are connected remains unclear. Previous study in Arabidopsis implied that priming with AHL depends on a stronger and prolonged activation of AtMPK6 (Schikora et al. 2011). In addition, exposure to oxo-C14-HSL and subsequent challenge with flg22, increased concentration of phenolics and lignin as well as callose depositions in Arabidopsis cell walls (Schenk et al. 2012; Schikora et al. 2011). Furthermore, accumulation of oxylipin in distal tissues during AHL-priming in Arabidopsis promoted stomatal closure. The closed stomata enhanced, consequently, plant resistance to bacterial pathogens (Schenk and Schikora 2014). The ability of barley to enhance resistance against pathogens, as a result of AHL-priming, was demonstrated previously by Hernandez-Reyes et al. (2014). The inoculation with the oxo-C14-HSL-producing E. meliloti strain improved the resistance against the powdery mildew-causing agent Blumeria graminis. Additionally, several quantitative trait loci (QTL) related to the resistance against powdery mildew were identified in barley (Bengtsson et al. 2017; Cantalapiedra et al. 2016; Douchkov et al. 2014; Romero et al. 2018; Silvar et al. 2013; Silvar et al. 2012). Given the principal ability of AHL-priming in barley and the differences in genetic background, Wehner et al. (2019) suggested that the effectiveness of oxo-C14-HSL-induced priming to enhance resistance against leaf rust (Puccinia hordei Otth.) is different in barley genotypes.

In this study, we investigated whether the differences in the genetic background of a genotype reflects on the capacity of priming for enhanced resistance against B. graminis f. sp. hordei and assessed the physiological basis of AHL-priming in barley. We hypothesized that the potential for priming is not a general feature but rather depends on the genotype. The enhanced resistance in different barley cultivars was assessed using two systems: the bacterial-based, in which the impact of the AHL-producing strain E. meliloti expR+ was compared with the influence of the AHLnegative E. meliloti attM strain, as well as a simpler system, in which we used the pure AHL molecule. We monitored the resistance toward B. graminis f. sp. hordei, the activation of MAP kinases, and expression of defense-related genes. Furthermore, we evaluated to what extent the principal mechanism of AHL-priming is similar in barley and Arabidopsis.

\section{MATERIALS AND METHODS}

Identification of genetically distant cultivars. In order to identify the genetically most diverse genotypes, a set of 64 sixrowed barley cultivars was genotyped with the 9k iSelect Chip (Comadran et al. 2012). Based on 3,212 polymorphic single nucleotide polymorphisms (SNP), filtered as described in Wehner et al. (2015), a modified Rogers Distance (MRD) matrix (Wright 1968) was calculated according to Reif et al. (2005) in R Studio (RStudio Team 2015). Based on these results a principal coordinate analysis (PCoA) was conducted (Gower 1967) using the stats package of $\mathrm{R}$, and five k-medoids were calculated (Kaufman and Rousseeuw 2005; Reynolds et al. 2006) with the prabclus package of R. Five genetically most diverse genotypes were identified and used in this study together with three reference genotypes.

Plant growth. The plants were grown in two different systems. For detached leaf assays and plant cell-conidia interaction studies, plants were grown on standard greenhouse substrate soil (Fruhstorfer Erde) for 2 weeks under greenhouse conditions at $18 / 16^{\circ} \mathrm{C}$ and 16/8 h (day/night) photoperiod. For gene expression analyses, metabolite profiling, assessment of MAP kinase activity and phenolic compounds that required controlled conditions and naïve plants, barley plants were grown under sterile conditions. Seeds were surface-sterilized by washing with sterile water, thrice for 1 min each. Subsequently, the seeds were submerged in $1.5 \%$ sodium hypochlorite for $20 \mathrm{~min}$ and then washed with sterile water thrice for $1 \mathrm{~min}$ each. The seeds were further washed with sterile water for $20 \mathrm{~min}$. Seeds were then placed on moist sterilized paper. After 5 days, equally developed seedlings were selected and three seedlings were grown in a jar containing $60 \mathrm{ml}$ of $1 / 2$ Murashige \& Skooge (MS) medium (50 ml of agar [0.8\%] + $10 \mathrm{ml}$ of broth). Plants were allowed to grow for an additional 3 days in controlled conditions: day/night $16 / 8 \mathrm{~h}$ and $18 / 16^{\circ} \mathrm{C}$ photoperiod, light intensity of $150 \mu \mathrm{mol} / \mathrm{m}^{2} \mathrm{~s}$ and $60 \%$ humidity, in a growth chamber.

Inoculation with Ensifer meliloti strains. Ensifer meliloti Rm2011 expR+ (M. McIntosh) and E. meliloti Rm2011 (pBBR2attM) carrying the lactonase gene attM from Agrobacterium tumefaciens (Zarkani et al. 2013) were grown in tryptone yeast (TY) extract medium until the $\mathrm{OD}_{600 \mathrm{~nm}}$ of 0.6 to 0.8 . Bacterial cultures were centrifuged at $2,500 \times g$ for $10 \mathrm{~min}$ and resuspended in $10 \mathrm{mM}$ $\mathrm{MgCl}_{2}$. The rhizosphere of soil-grown barley was inoculated three times over 2 weeks with $10 \mathrm{ml}$ of $\mathrm{OD}_{600 \mathrm{~nm}}=0.1$ using E. meliloti

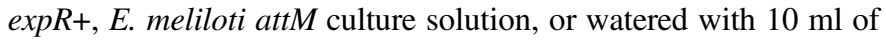
$10 \mathrm{mM} \mathrm{MgCl}{ }_{2}$ as control. The production of $\mathrm{AHL}$ on the root surface as well as establishment of the bacteria was previously demonstrated by Zarkani et al. (2013).

Challenge with $\boldsymbol{B}$. graminis f. sp. hordei. Three days after the last inoculation with E. meliloti, $\mathrm{MgCl}_{2}$, or with the pure oxo-C14HSL, or the solvent control acetone in case of the in vitro assay, second leaves were placed on water agar plates (1\%) with adaxial 
side of leaves faced up. The leaves were challenged with fresh $B$. graminis f. sp. hordei conidial spores, approximately 100 conidia $/ \mathrm{cm}^{2}$, using infected barley plants. The powdery mildew fungus B. graminis (DC) Speer f. sp. hordei EM Marchal, race A6, was maintained on the cultivar Golden Promise under the conditions described above. The plates containing the infected leaves were kept at room temperature with low light conditions for 2 days for plant cell-conidia interaction assay and for 5 days for pustules count. The pustules on the infected leaves were counted per square centimeter using a stereomicroscope.

Stain with 3,3-diaminobenzidine (DAB) and plant cell-conidia interaction assay. Two days after the challenge with B. graminis f. sp. hordei, leaves were partially submerged overnight in DAB solution ( $\mathrm{pH} \mathrm{3.8)}$ at a concentration of $1 \mathrm{mg} / \mathrm{ml}$. Subsequently, the leaves were submerged for 2 days in a de-staining solution (ethanol/ chloroform/trichloroacetic acid [4:1:0.0075]) and stored in 50\% glycerol until further analysis. Ink blue containing acetic acid was used for staining B. graminis f. sp. hordei. A minimum of 200 different cell-conidia interactions from each leaf were analyzed using an Axioplan 2 microscope (Zeiss, Germany) and each interaction was assigned to one of the following: (i) elongated secondary hyphae (ESH) development, (ii) papillae formation, or (iii) hypersensitive response (HR). The percentage of occurrence of each outcome was calculated subsequently.

Pretreatment with oxo-C14-HSL. Plants grown for 3 days under sterile and controlled conditions were pretreated with the priming agent $\mathrm{N}$-(3-oxotetradecanoyl)-L-homoserine lactone (oxoC14-HSL) (Schikora et al. 2011). Oxo-C14-HSL (Sigma-Aldrich) was dissolved in acetone to acquire a stock solution of $60 \mathrm{mM}$. Barley seedlings on 1/2 MS medium under sterile conditions were pretreated with oxo-C14-HSL for 3 days at a final concentration of $6 \mu \mathrm{M}$. Oxo-C14-HSL was added in a reservoir containing liquid $1 / 2$ MS medium in the middle of the jar. Thereafter, plants were grown for an additional 3 days in the same conditions (day/night 16/8 h photoperiod, $18 / 16^{\circ} \mathrm{C}$, light intensity of $150 \mu \mathrm{mol} / \mathrm{m}^{2} \mathrm{~s}$, and $60 \%$ humidity). All experiments were performed with the solvent control acetone.

Treatment with chitin. One gram of chitin (Sigma-Aldrich) was dissolved in $500 \mu \mathrm{l}$ of $96 \%$ acetic acid and filled up to $50 \mathrm{ml}$ with $\mathrm{H}_{2} \mathrm{O}$ (MilliQ) in order to obtain a final concentration of $20 \mathrm{mg} / \mathrm{ml}$. The solution was subsequently stirred for $10 \mathrm{~min}$ and then autoclaved at $121^{\circ} \mathrm{C}$ for $15 \mathrm{~min}$, followed by centrifugation at $2,000 \times g$ for $5 \mathrm{~min}$. Considering that about one-fifth of total chitin dissolved in water containing acetic acid, the centrifuged chitin concentration was estimated at $4 \mathrm{mg} / \mathrm{ml}$. The solution was further diluted in order to obtain a final concentration of $1 \mathrm{mg} / \mathrm{ml}$. One milliliter of chitin solution $(1 \mathrm{mg} / \mathrm{ml})$ was pipetted into each jar.

Gene expression analyses. Roots of in vitro-grown barley plants, genotypes Golden Promise (GP) and Gaulois pretreated with oxo-C14-HSL, were harvested at $0,6,24,48$, and $72 \mathrm{~h}$ post chitin $(100 \mu \mathrm{g} / \mathrm{ml})$ treatment. The root material was homogenized and total RNA was extracted using spin columns (RNeasy Plant mini kit, Qiagen). RNA concentration and quality were determined using the Nanodrop Bioanalyzer. One microgram of total RNA was DNAsedigested using DNAse kit (Quantabio) and subsequently cDNA synthesis was carried out using the Quantabio kit protocol according to manufacturer's recommendations (qScript cDNA Synthesis Kit [Quantabio]). Quantitative RT-PCR (qPCR) was performed using primers listed in Supplementary Table S1. All expression levels were normalized to the expression of $H v U B Q 60$ (GenBank M60175.1).

Assessment of MAP kinase activity. Root samples from in vitro-grown plants and pretreated for 3 days with $6 \mu \mathrm{M}$ oxo-C14HSL or acetone (control) were collected at 0, 45, 90, and $135 \mathrm{~min}$ after treatment with chitin. Proteins were extracted from homogenized barley roots using Tris-based buffer $(25 \mathrm{mM}$ Tris- $\mathrm{HCl}$ at $\mathrm{pH}$ 7.8, $75 \mathrm{mM} \mathrm{NaCl}, 15 \mathrm{mM}$ EDTA, $10 \mathrm{mM} \mathrm{MgCl}_{2}$, and $1 \mathrm{mM}$ DTT) supplemented with a complete protease inhibitor cocktail and a phosphatase inhibitor cocktail (Roche). The homogenized root samples were vortexed rigorously and left for $5 \mathrm{~min}$ on ice and subsequently centrifuged at $14,000 \mathrm{rpm}$ at $4^{\circ} \mathrm{C}$ for $15 \mathrm{~min}$. The protein concentration of the cell lysates was determined using Bradford assay (5x Roti Quant, Roth). Thirty micrograms of total protein was run on three sodium dodecyl sulfate gels (12\%) concomitantly and two of them were transferred to PVDF membrane through semiwet blotting protocol. The membranes were blocked with $2 \%$ bovine serum albumin (BSA) and thereafter probed with respective primary antibodies: $\alpha$ AtMPK6 (Sigma-Aldrich) or $\alpha p E R K 1 / 2$ (Cell Signaling), followed by incubation with horseradish peroxidase-labeled secondary antibody. Blots were developed using ServaLight chemiluminescent substrate (SERVA). The third gel was stained with Coomassie Brilliant Blue.

Assessment of phenolic compounds. Whole phenolic compounds were measured via a colorimetric assay based on the FolinCiocalteu method (Eynck et al. 2009) with some modifications. Whole in vitro-grown barley plants, Golden Promise or Gaulois, pretreated with $6 \mu \mathrm{M}$ oxo-C14-HSL and treated with chitin $(100 \mu \mathrm{g} / \mathrm{ml})$ were harvested at 0 and $48 \mathrm{~h}$ post chitin treatment, homogenized and lyophilized. A dry mass of $30 \mathrm{mg}$ of lyophilized plant tissue was used to measure the concentration of different phenolic compounds and lignin. Soluble phenolics were extracted using $80 \%$ aqueous methanol and quantified using the Folin-Ciocalteu assay (Ainsworth and Gillespie 2007) and gallic acid (Sigma-Aldrich, Darmstadt, Germany) as a reference for quantification. Subsequently, following a slightly modified protocol by Schenk and Schikora (2015), alkaline hydrolysis of dried pellet was carried out using $1 \mathrm{M} \mathrm{NaOH}$ at $80^{\circ} \mathrm{C}$ overnight and ethyl acetate was further added to the pellets to dissolve the cell wall-bound phenolic compounds. The absorbance for both soluble and cell wall-bound phenolic compounds was measured at $765 \mathrm{~nm}$. Subsequently, samples were washed several times with acetone and dried in the SpeedVac for $10 \mathrm{~min}$. Pellets were used for lignin quantification using the thioglycolic acid method (Schenk and Schikora 2015).

Metabolite profiling of plant extracts. Barley plants, genotypes Golden Promise and Gaulois, grown in vitro and pretreated with $6 \mu \mathrm{M}$ oxo-C14-HSL or acetone, were challenged with chitin $(100 \mu \mathrm{g} /$ $\mathrm{ml}$ ) and whole plants were harvested at 0 and $48 \mathrm{~h}$ post challenge. Three independent pools of plants per experimental variant were used for metabolite profiling. Shock frozen plants were homogenized in liquid nitrogen using mortar and pestle. Two aliquots of $50 \mathrm{mg}$ $( \pm 2.5 \mathrm{mg})$ per sample were weighed into precooled 2-ml tubes and placed in a precooled $\left(-80^{\circ} \mathrm{C}\right)$ rack. Four hundred microliters of precooled methanol/water at $-80^{\circ} \mathrm{C}, 80 / 20$ (vol/vol), was added and samples were immediately vortexed and slowly thawed under periodic vortexing. After reaching room temperature, 400 pmol biochanin A (Sigma-Aldrich) was added as surrogate standard. The samples were sonicated (15 min, room temperature), shaken (30 min, room temperature, $2,400 \mathrm{~min}^{-1}$ ), and centrifuged (10 $\mathrm{min}$, room temperature, $13,000 \times g$ ). The supernatants were collected and the remaining pellets extracted for a second time using $400 \mu \mathrm{l}$ of methanol/water, 80/20 (vol/vol), as described above. The combined supernatants were evaporated to dryness using a vacuum centrifuge $\left(10 \mathrm{mbar}, 30^{\circ} \mathrm{C}\right)$. The remaining residues were redissolved in $400 \mu \mathrm{l}$ of methanol/water, 50/50 ( $\mathrm{vol} / \mathrm{vol})$, sonicated (10 min, room temperature), and centrifuged (10 $\mathrm{min}$, room temperature, 13,000 $\times g$ ). The resulting supernatants were transferred into vials and subjected to liquid chromatography/mass spectrometry (LC/MS)-based metabolite profiling as was done in Böttcher et al. (2017, 2018). 
Raw data files were converted into mzData format using MassHunter Qualitative Analysis software (Agilent Technologies), arranged according to genotype and treatment in eight sample classes and processed individually for each ion mode using the $\mathrm{R}$ package XCMS (Smith et al. 2006). Feature detection was performed using the centWave algorithm (parameters: sntresh $=3$, prefilter $=(3,1000), \mathrm{ppm}=25$, peak width $=(5,12))$. Alignment was accomplished using the group.density function (parameters: minfrac $=2 / 3, \mathrm{bw}=1.5, \mathrm{mzwid}=0.02$ ). Missing values were replaced by evenly distributed random numbers in the range of 300 to 500 as estimated intensity threshold. Prior to multivariate analysis, all feature intensities were normalized by fresh weight, averaged for the two technical replicates, and $\log _{2}$ transformed. Principal component analyses were performed using stats package of $\mathrm{R}$ Studio (RStudio Team 2015).

Statistical analysis. All experiments were performed with at least three independent biological replicates. The GENMOD procedure from SAS 9.4 (SAS Institute Inc., Cary, NC) was used for the analysis of variance. Class variable was treatment (E. meliloti expR+, E. meliloti attM, and $\mathrm{MgCl}_{2}$ solvent control). For multiple comparisons, the $P$ value was adjusted by the method of Tukey's honestly significant difference post hoc test. In the box plot data representation, boxes represent interquartile range and the ends of the boxes represent the upper and lower quartile range $(50 \%)$. The whiskers represent the lowest and highest range of data. Quantitative PCR assays were performed in three biologically independent experiments, and $P$ values $<0.05$ in the Student's $t$ test were considered as indicative for significant difference. Western blot analysis was performed in three independent experiments, and representative blot is shown. Graphs were made using Prism 7 (GraphPad Software, La Jolla, CA).

\section{RESULTS}

Barley genotypes have distinct abilities to enhance resistance against B. graminis. To verify our hypothesis that the potential for priming is not a general feature but rather depends on the genotype, we first determined genetically distant genotypes in a 64 set of sixrowed barley (Hordeum vulgare L.) cultivars (Supplementary Fig. $\mathrm{S} 1$ ). Leaves from soil-grown plants of five identified genotypes Hasso, Gaulois, Carola, Plana, and Hampus, together with Golden Promise, Otto, and Igri, used as reference genotypes, were used for detached leaf assay in order to assess the differences in resistance against $B$. graminis f. sp. hordei. We observed that the genotypes had different native resistance (Supplementary Fig. S2). Genotypes like Golden Promise and Igri were susceptible and allowed high level of fungal growth, whereas Carola was relatively resistant toward this fungus. The five other genotypes (Igri, Hasso, Gaulois, Otto, and Plana) had intermediate resistance.

In a next step, we inoculated soil-grown plants with the oxo-C14HSL-producing strain E. meliloti expR+, E. meliloti strain attM, which is not able to accumulate the AHL and $\mathrm{MgCl}_{2}$ as solvent control prior to the B. graminis f. sp. hordei challenge. Although the inoculation had no impact on plant's fresh or dry weight (Supplementary Fig. S3), we were able to observe that the bacterial strains had different impact on the resistance of tested genotypes against $B$. graminis f. sp. hordei (Fig. 1A). The analyzed genotypes presented three distinct response patterns. The first group included Golden Promise and Igri. Both genotypes reacted to the inoculation with $E$. meliloti with induced resistance, as suggested by the lower number of pustules after inoculation with E. meliloti attM. Moreover, their defense mechanisms could be enhanced even further by the presence of AHL, as observed after inoculation with E. meliloti strain expR+ (Fig. 1B). The second group included
Hasso, Otto, and Plana, those genotypes responded with enhanced resistance only after inoculation with the AHL-producing strain E. meliloti expR+. The third group includes Gaulois and Hampus. Those genotypes were nonresponsive to inoculation with either E. meliloti strain (Fig. 1A and 1B). Taking together, five out of eight tested genotypes responded positively to AHL-priming although, the capacity varied among them.

Interactions between barley cells and $B$. graminis f. sp. hordei conidia reflect only partially the macroscopic response. To further substantiate the observed phenotypes, we analyzed the interaction between the plant host and the fungal pathogen on a cellular level assessing the plant cell-conidia interaction on detached leaves from soil-grown plants inoculated and challenged with $B$. graminis $\mathrm{f}$. sp. hordei as described above. The development of ESH was significantly different among the genotypes and among the treatments. Inoculation with the oxo-C14-HSL-producing strain E. meliloti $\exp R+$ resulted in lower frequency of elongated secundary hyphae (ESH) formation compared with the control $\left(\mathrm{MgCl}_{2}\right)$ treatment, in all genotypes except for Gaulois (Fig. 2A). The inoculation of barley genotypes with the lactonase-expressing strain E. meliloti attM had no significant effect on the ESH occurrence. The only exception was Igri, where we observed reduction in the ESH incidence. Our results revealed that the oxo-C14-HSL produced by E. meliloti expR+ strain resulted in higher resistance of the plants since seven of the eight tested genotypes were able to inhibit the ability of $B$. graminis $\mathrm{f}$. sp. hordei conidia to form secondary hyphae. This effect was not observed in the Gaulois genotype (Fig. 2A and D).

The frequency of the two other categories: formation of papillae and hypersensitive response (HR) (Fig. 2E and F), which reveal resistance, was analyzed subsequently. The rate of papillae formation was not influenced by the inoculation with E. meliloti attM (Fig. 2B), except for Plana in which it was reduced. The inoculation with the oxo-C14-HSL-producing strain E. meliloti expR+ had positive impact (higher percentage of papillae) on Golden Promise, Igri, Hasso, and Otto, whereas it had no impact on Gaulois, Plana, Hampus, and Carola (Fig. 2B). In analogy to the papillae occurrence, plants inoculated with the oxo-C14-HSL-producing

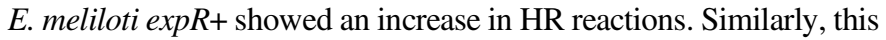
response was not detected in Gaulois. Notably, plants inoculated with the attM strain did not significantly differ from the control plants among all genotypes. Taking together, those results suggest that AHL impact on defense depends on the genetic background, and genotypes like Golden Promise respond very well to the AHL as priming agent while the genotype Gaulois is not able to induce such priming.

Impact of AHL on resistance to $B$. graminis f. sp. hordei and reactive oxygen species (ROS) accumulation differ between Golden Promise and Gaulois genotypes. To corroborate those finding even further, we performed a detached leaf assay on in vitrogrown Golden Promise, since it responds very well to the AHL as a priming agent and Gaulois, not able to induce AHL-priming. The plants were exposed to either $6 \mu \mathrm{M}$ oxo-C14-HSL or the solvent control (acetone) and challenged with B. graminis f. sp. hordei. Comparison between the experimental outcomes revealed that in the Golden Promise genotype, pretreatment with AHL lowered the susceptibility toward B. graminis f. sp. hordei. This effect was not observed in the Gaulois cultivar (Fig. 3A).

In addition, we observed accumulation of ROS after a challenge with B. graminis f. sp. hordei. The stronger accumulation of ROS, observed in oxo-C14-HSL pretreated Golden Promise plants after B. graminis f. sp. hordei challenge was missing in Gaulois (Fig. 3B).

Activation of MAP kinases and expression of defense-related genes. To examine whether the priming in barley is associated with a stronger activation of MAP kinases, we carried out a western blot-based kinase assay on in vitro-grown plants pretreated with 
$6 \mu \mathrm{M}$ oxo-C14-HSL and subsequently treated with chitin. The results showed that plants perceived chitin as elicitor and transiently activated MAPKs. In acetone pretreated Golden Promise, the MAPK activity was increased 45 and 90 min after chitin treatment and returned to nearly basal level $135 \mathrm{~min}$ after chitin treatment (Fig. 4). In contrary, upon oxo-C14-HSL pretreatment, Golden Promise showed stronger and prolonged activation of MAPKs. Evident and prominent activation was observed even 135 min after chitin treatment. In the genotype Gaulois, we observed different activation pattern of the MAPKs, the activation level was high at 45 min after the chitin treatment; however, in both treatments, it decreased 135 min after the treatment with chitin (Fig. 4A).

To verify if pretreatment with AHL alters the transcriptional activation of defense-related genes, we performed qPCR analysis from RNA extracted from the roots at different time points of Golden Promise and Gaulois plants before and after chitin challenge in plants pretreated with oxo-C14-HSL or acetone (control). Analysis of Pathogenesis-Relatedl (PRI) expression in Golden
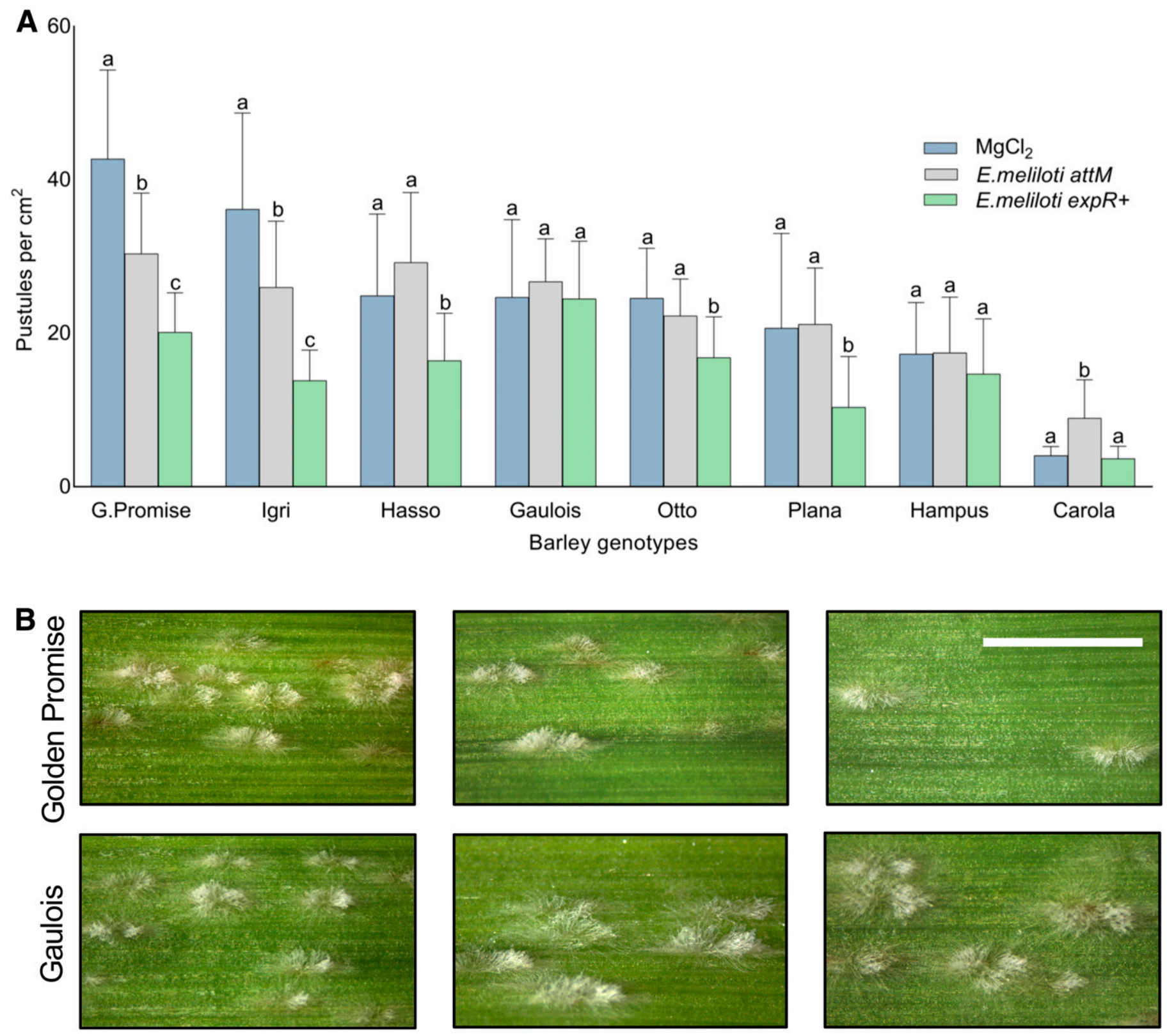

$\mathrm{MgCl}_{2}$

attM
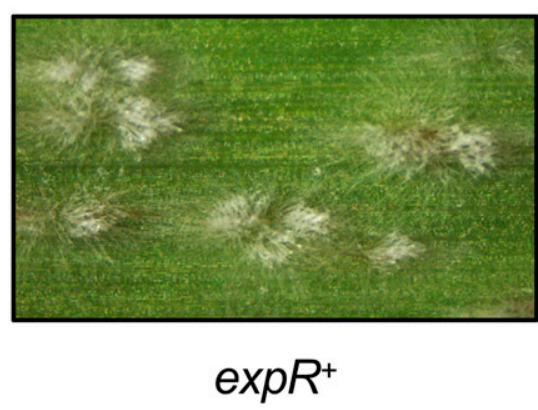

Fig. 1. Inoculation with Ensifer meliloti influences resistance only in particular barley genotypes. A, Eight different genotypes of barley growing on soil were inoculated three times over 2 weeks with $10 \mathrm{ml}$ of $10 \mathrm{mM} \mathrm{MgCl}_{2}$ (control), $10 \mathrm{ml}$ of $0.1 \mathrm{OD}_{600 \mathrm{~nm}}$ of the lactonase-expressing strain $E$. meliloti attM, or $10 \mathrm{ml}$ of $0.1 \mathrm{OD}_{600 \mathrm{~nm}}$ of the oxo-C14-HSL-producing strain E. meliloti expR+. Second leaves of inoculated plants were placed on water agar plates and subsequently challenged with Blumeria graminis f. sp. hordei (100 conidial spores $\left./ \mathrm{cm}^{2}\right)$. Pustules were counted 5 days post challenge. The bar represents mean and standard deviation of at least 10 biological replicates from four independent experiments. Different letters indicate $P \leq 0.05$ in Tukey's honestly significant difference post hoc test. B, Representative photos of leaves infected with B. graminisf. sp. hordei of two cultivars: the $N$-acyl homoserine lactone (AHL)-primable Golden Promise and the AHL-nonprimable Gaulois. Photos were taken 7 days after challenge with conidial spores. The pretreatment was performed as indicated. Scale bar indicates $0.5 \mathrm{~cm}$. 

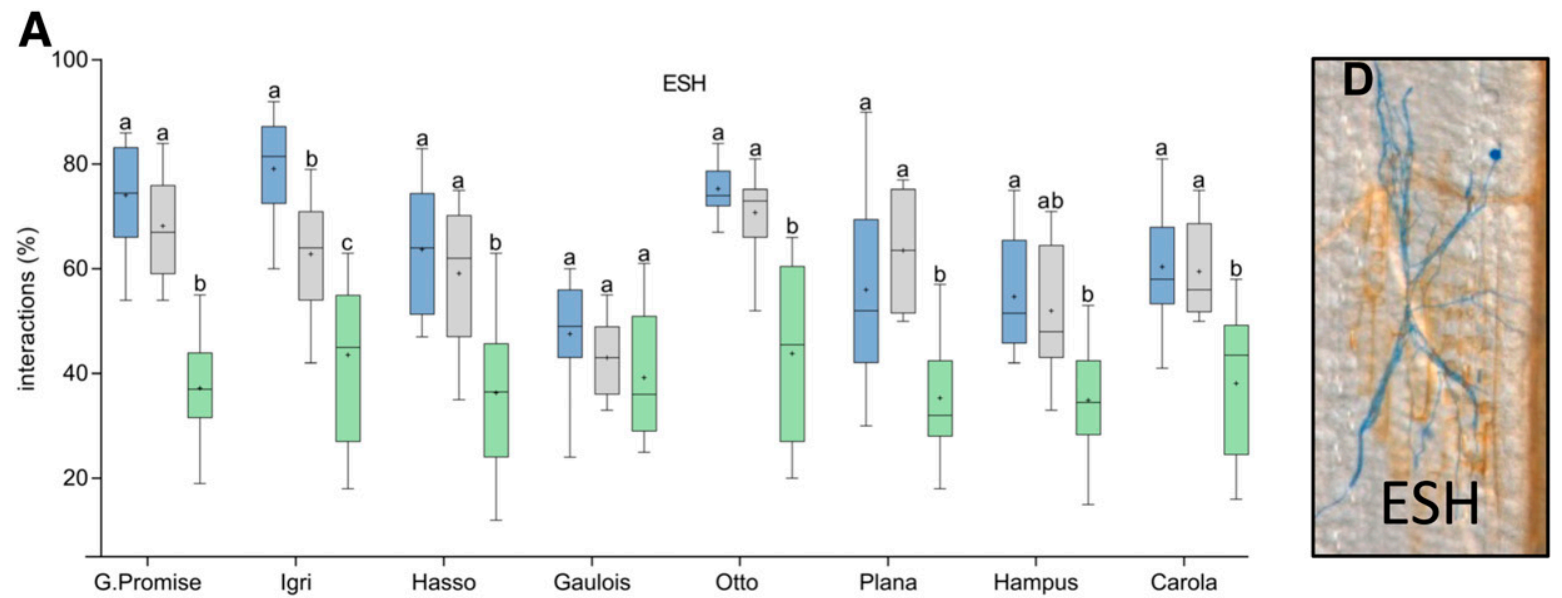

B
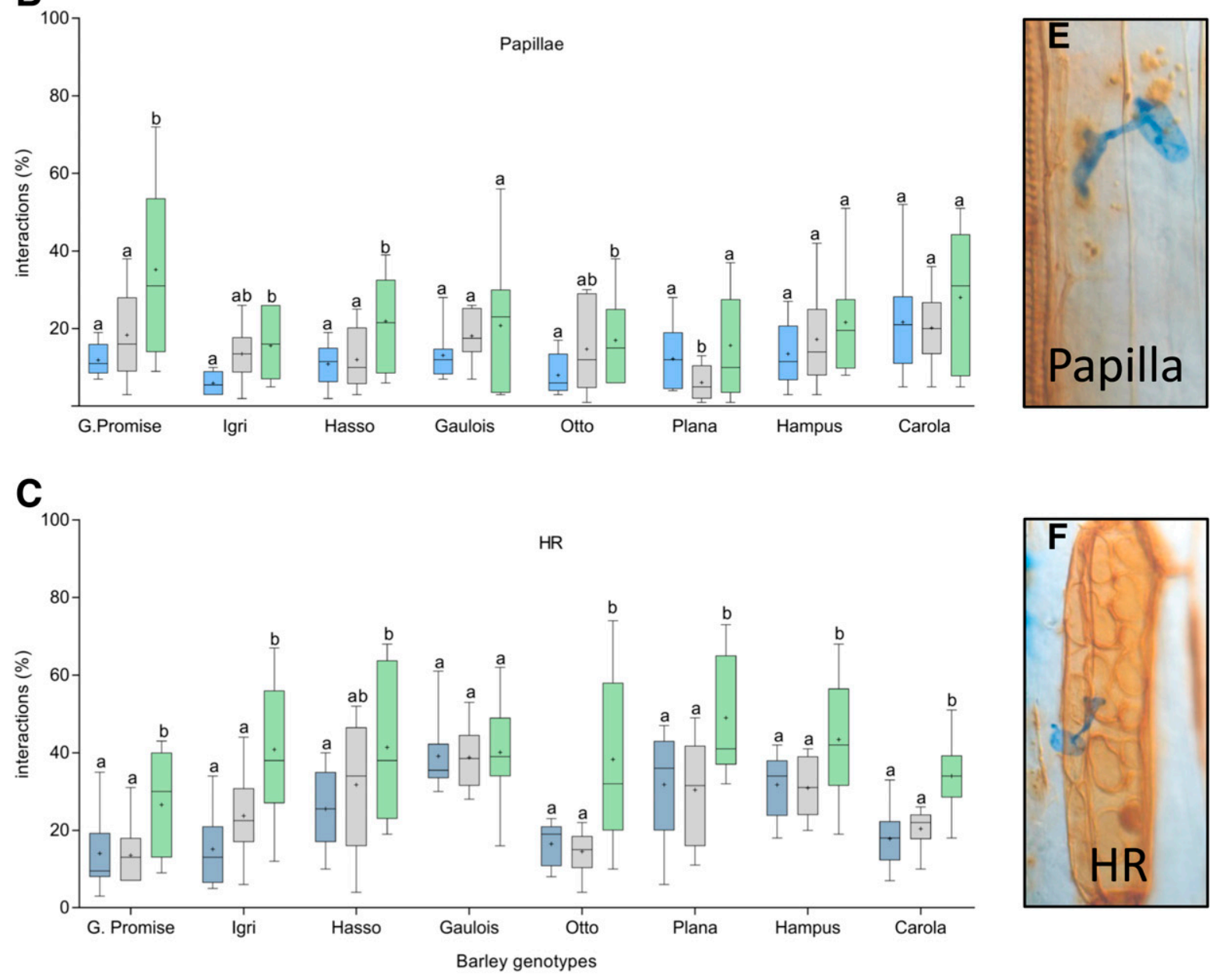

$\square \mathrm{MgCl}_{2} \square$ E. meliloti attM $\square$ E. meliloti expR+

Fig. 2. Response of barley cells to Blumeria graminis f. sp. hordei. Incidence of A, elongated secondary hyphae (ESH), B, papillae, or C, hypersensitive response (HR) after $B$. graminis f. sp. hordei challenge. Plants from eight genotypes that were grown on soil and inoculated with $10 \mathrm{ml}$ of $10 \mathrm{mM} \mathrm{MgCl} 2$ (control), $10 \mathrm{ml}$ of $0.1 O . D_{600 \mathrm{~nm}}$ of the lactonase-expressing strain $E$. meliloti attM, or $10 \mathrm{ml}$ of $0.1 \mathrm{OD}_{600 \mathrm{~nm}}$ of $E$. meliloti expR+ strain, which produces substantial amount of oxo-C14-HSL. Second leaves of inoculated plants were cut and placed on a water plate agar and challenged with $B$. graminisf. sp. hordei spores (100 conidial spores $/ \mathrm{cm}^{2}$ ). The percentage of interaction sites resulting in either ESH or mycelium demonstrating susceptibility against the A, pathogen, B, papillae, or $\mathbf{C}, \mathrm{HR}$ indicating resistance was assessed 2 days after challenge. Experiment was repeated four times. Small letters indicate differences in the Tukey test at $P<0.05$. Boxes represent interquartile range and the ends of the boxes represent the upper and lower quartile range $(50 \%)$. The whiskers represent the lowest and highest range of data and + sign represents mean of data. Adjacent to the graphs are shown representative photographs demonstrating the possible results of interaction between barley leaf cells and $B$. graminis f. sp. hordei. From top: the formation of $\mathbf{D}, \mathrm{ESH}, \mathbf{E}$, papillae, and $\mathbf{F}$, HR. 
Promise revealed higher expression level in AHL pretreated plants, even before the challenge. Moreover, when compared with control, oxo-C14-HSL pretreated plants exhibit 13-fold increase in the expression level, $72 \mathrm{~h}$ post chitin treatment (Fig. 4B). Similarly, $P R 17 b$ gene expression was 12-fold higher in oxo-C14-HSL pretreated Golden Promise, even before chitin treatment. Furthermore, 48 and $72 \mathrm{~h}$ post chitin treatment the expression was higher in oxoC14-HSL pretreated plants (Fig. 4D). Contrary to Golden Promise, there was no considerable difference in the expression of $P R I$ and $P R 17 b$ between the two treatments in Gaulois; prior and after the chitin treatment (Fig. 4C and E). Similar, however less pronounced tendencies were observed for CSD1, Hsp70, Prx7, and GSL7 genes (Supplementary Fig. S4).

Priming in barley is reflected through distinct accumulation of specific metabolites. In order to ascertain changes in metabolites, we performed a nontargeted metabolite profiling approach. Methanolic extracts of whole seedlings grown in vitro, pretreated with $6 \mu \mathrm{M}$ oxo-C14-HSL and treated with chitin, were used. Overall, 5,590 and 2,472 molecular features (mass-over-chargeretention time pairs) were detected and relatively quantified in positive and negative ion mode, respectively (Supplementary Table S2). Principal component analysis (PCA) of metabolite profiles acquired in positive ion mode revealed a clear separation of the two cultivars along PC 1 accounting for $50.9 \%$ of the total variation (Fig. 5A). A partial discrimination according to chitin treatment is associated with PC2 which accounts for $6.4 \%$ of the total variance. PCA of the metabolite profiles acquired in negative ion mode gave a very similar picture: $47.4 \%$ of the total variance is represented by PC1 and attributed to genotype specific differences, whereas PC2 (5.4\%) partly reflects metabolic variation upon chitin treatment (Fig. 5C). Interestingly, detailed and pairwise comparison of the abundance of individual molecular features between the different sample classes revealed more features significantly accumulated in Golden Promise after oxo-C14-HSL treatment and subsequent chitin challenge than in Gaulois: 85 versus 28 (positive ion mode) and 40 versus 17 (negative ion mode). The nature of these compounds is currently not known.

Phenolic cell wall components accumulate in Golden Promise but not in Gaulois. Subsequently, we quantified different phenolic compounds in the Golden Promise and Gaulois genotypes before and after chitin treatment in plants grown in vitro and pretreated with $6 \mu \mathrm{M}$ oxo-C14-HSL or acetone (control). The two tested barley genotypes differed considerably in regard to phenolic compounds and lignin accumulation (Fig. 6). The colorimetric analysis of soluble and cell wall-bound phenolic compounds in Golden Promise revealed significant increase in accumulation in seedlings pretreated with oxo-C14-HSL compared with control plants (Fig. 6A and B). This effect was observed in the seedlings of Golden Promise before and after chitin treatment. In contrast, irrespective of oxo-C14-HSL pretreatment or time after chitin treatment, the amount of soluble and cell wall bound phenolic compounds was not changed in Gaulois seedlings (Fig. 6D and E). Furthermore, we observed significant increase in lignification in seedlings pretreated with oxo-C14-HSL and treated for $48 \mathrm{~h}$ with chitin $(P<0.004)$, whereas there was no difference before chitin treatment $(P>0.05)$ (Fig. 6C). On the other hand, Gaulois did not show any significant increase in lignin accumulation in their seedling tissues after pretreatment with oxo-C14-HSL, if compared with acetone control at 0 or $48 \mathrm{~h}, P=0.722$ and 0.597 , respectively.

\section{DISCUSSION}

This study aimed to assess the differences in the AHL-priming capacity between different genotypes of barley. We revealed that barley genotypes not only vary in the resistance against $B$. graminis f. sp. hordei, also the capacity to respond to bacterial QS molecule differs. Together with the recent study (Wehner et al. 2019), we suggest therefore that priming for enhanced resistance induced by oxo-C14-HSL is genotype-dependent. Among different barley accessions, we identified so called "AHL-primable" genotype that had the ability to enhance resistance and "AHL-non-primable" genotype that was nonresponsive to oxo-C14-HSL and showed no ability to enhance resistance after an AHL-priming.

Upon recognition of $B$. graminis f. sp. hordei, barley cells initiate formation of papillae (cell wall appositions) at the site of penetration attempt, which is one of the earliest plant defense responses at a cellular level (Carisse et al. 2000; Zeyen et al. 2002). We observed that the genotypes that had no improved ability to inhibit fungal growth after the AHL-priming, also did not exhibit higher percentage of papillae after inoculation with

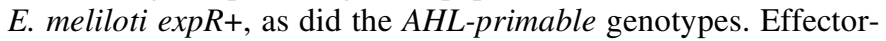
triggered immunity response usually deploys a defense mechanism that involves rapid oxidative burst due to high production of ROS (Heller and Tudzynski 2011; O'Brien et al. 2012). This leads to enhanced resistance in the form of localized cell death at the site of fungal penetration termed HR, which inhibits further fungal development (Huckelhoven and Kogel 2003). Very interesting

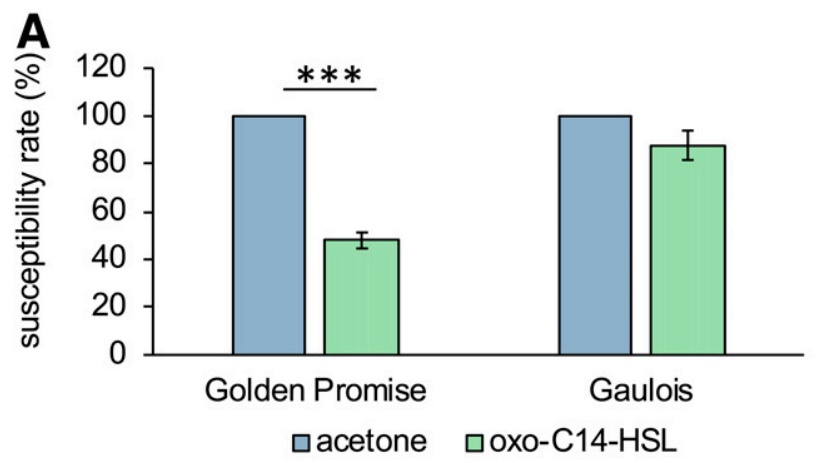

\section{B Golden Promise}

Gaulois

$48 \mathrm{~h}$ after Bgh challenge

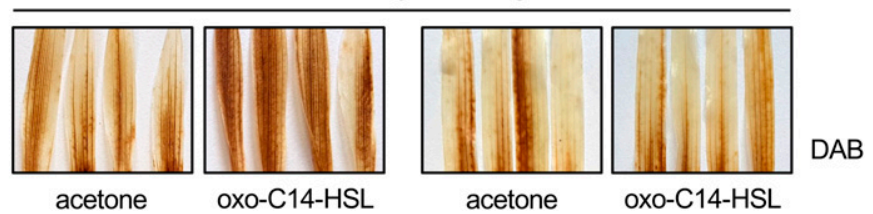

Fig. 3. Resistance to Blumeria graminis f. sp. hordei (Bgh) and reactive oxygen species (ROS) accumulation in oxo-C14-HSL pretreated plants. A, Barley plants were grown in vitro and pretreated with $6 \mu \mathrm{M}$ oxoC14-HSL or acetone (solvent control). The second leaves were placed on water agar plates and subsequently challenged with $B$. graminis f. $\mathrm{sp}$. hordei (100 conidial spores $/ \mathrm{cm}^{2}$ ). Pustules were counted 5 days post challenge. The results represent susceptibility ratio between acetone control (100\%) and oxo-C14-HSL pretreated plants. Error bars represent standard deviation from three independent biological repetitions. $n=25$; ${ }^{\star \star \star}$ indicates $P \leq 0.0005$ in Student's $t$ test. B, ROS accumulation in leaves from plants pretreated with $6 \mu \mathrm{M}$ oxo-C14-HSL or acetone (control) for 3 days and thereafter challenged with $B$. graminis f. sp. hordei. Both barley genotypes were grown under sterile condition. Second leaves of treated plants were placed on water agar plates and subsequently challenged with the $B$. graminis $\mathrm{f}$. $\mathrm{sp}$. hordei (100 conidial spores $/ \mathrm{cm}^{2}$ ). After 2 days of challenge, leaves were stained with DAB overnight and then de-stained to visualize the accumulation of ROS. 


\section{A Golden Promise}

\section{Gaulois}
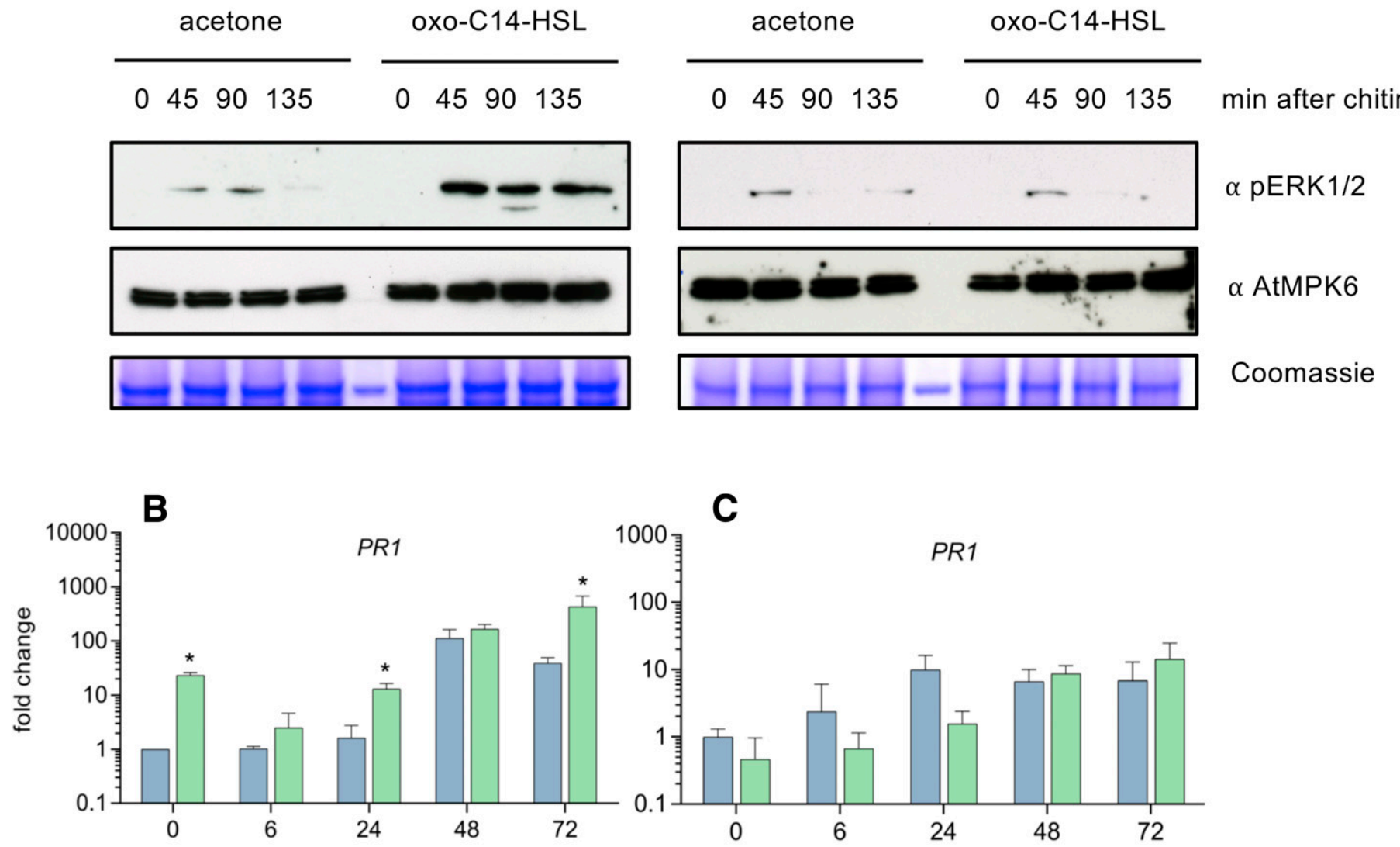

D

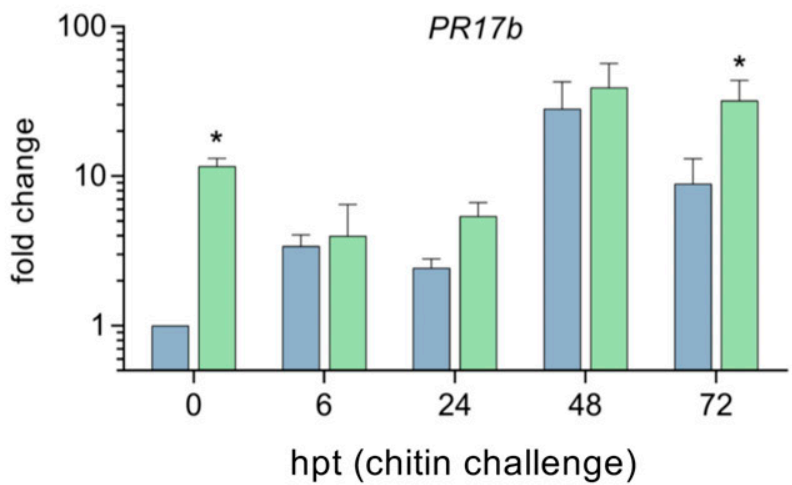

E

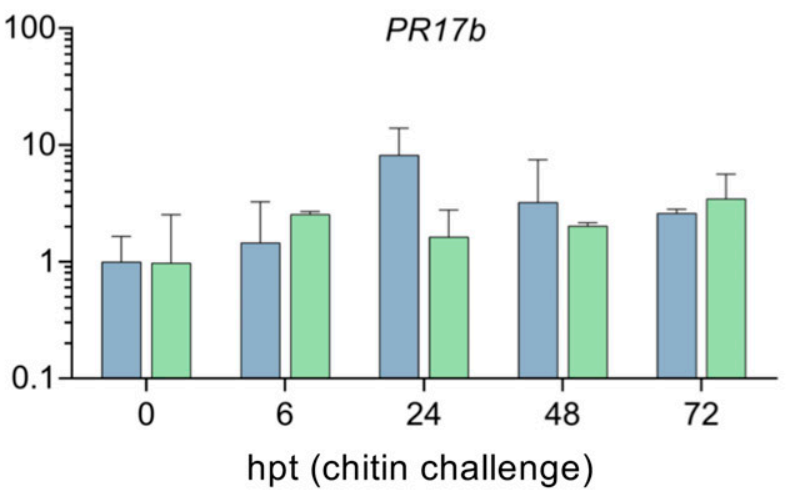

acetone

oxo-C14-HSL

Fig. 4. MAPK activation and expression of defense related genes. A, Two barley genotypes, Golden Promise and Gaulois, were grown under sterile condition and pretreated for 3 days with $6 \mu \mathrm{M}$ oxo-C14-HSL or acetone (solvent control), before treating with chitin (100 $\mu \mathrm{g} / \mathrm{ml})$. The plants were harvested as indicated over the time span of $135 \mathrm{~min}$. Roots of the treated plants were homogenized and protein was extracted. Thirty micrograms of protein was run on three sodium dodecyl sulfate gels (12\%) concomitantly. Activation of the barley MAP kinase was detected by probing with an $\alpha$ pERK1/ 2 antibody and the presence of the MAPK was detected with an $\alpha$ AtMPK6 antibody. Equal loading of proteins was confirmed by staining with Coomassie Brilliant blue. B to E, Expression profile of two defense-related genes $P R 1$ and $P R 17 b$ was monitored at various time points post treatment with chitin $(100 \mu \mathrm{g} / \mathrm{ml})$ in B and D, Golden Promise and C and E, Gaulois. Plants were pretreated with $6 \mu \mathrm{M}$ oxo-C14-HSL or acetone (solvent control) prior to chitin treatment. The abundance of each gene transcript in plant roots was normalized with ubiquitin transcript and 0 hours post treatment (hpt) levels. The bar represents mean of three biological replicates. Error bars represent standard deviation from three independent biological repetitions. * indicates $P<0.05$ in Student's $t$ test. 
was the fact that if compared with inoculation with the lactonaseexpressing E. meliloti attM strain or the $\mathrm{MgCl}_{2}$ control, after inoculation with E. meliloti $\exp R+$ all genotypes increased the proportion of HR observed after challenge with B. graminis f. sp. hordei (Fig. 2C). Solely, the nonresponsive genotype Gaulois exhibited unchanged response. The comparison of the Golden Promise and Gaulois on the SNP level revealed the dissimilarity between the two genotypes (Supplementary Fig. S5). These as
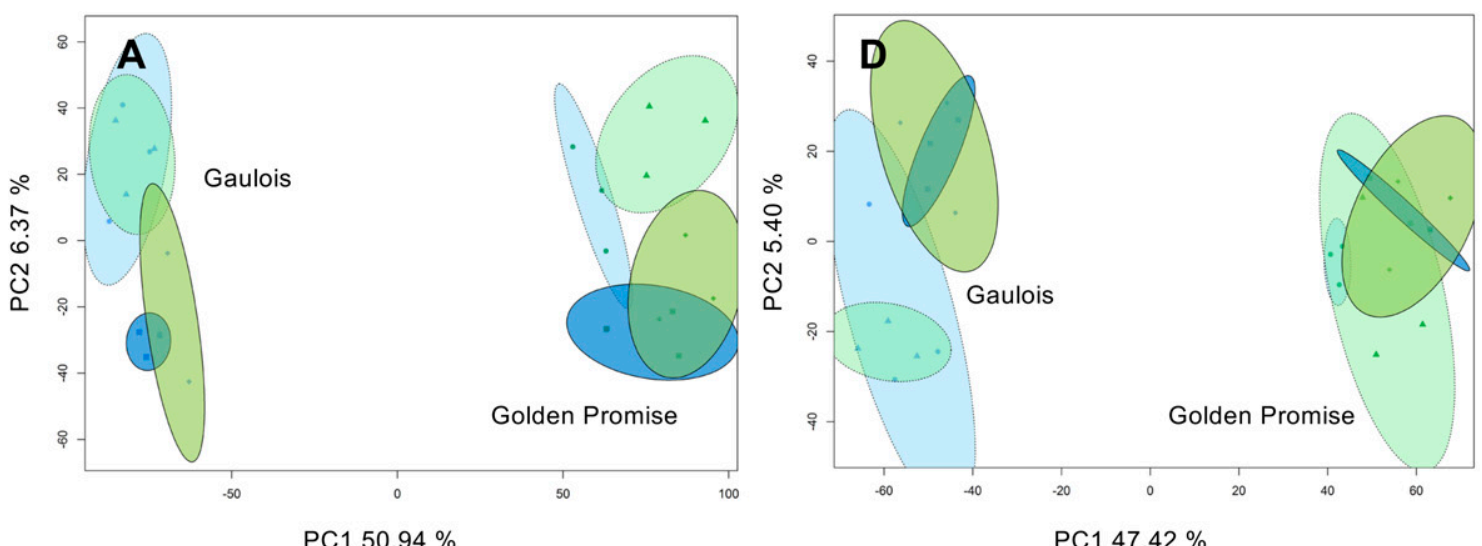

\section{Golden Promise}
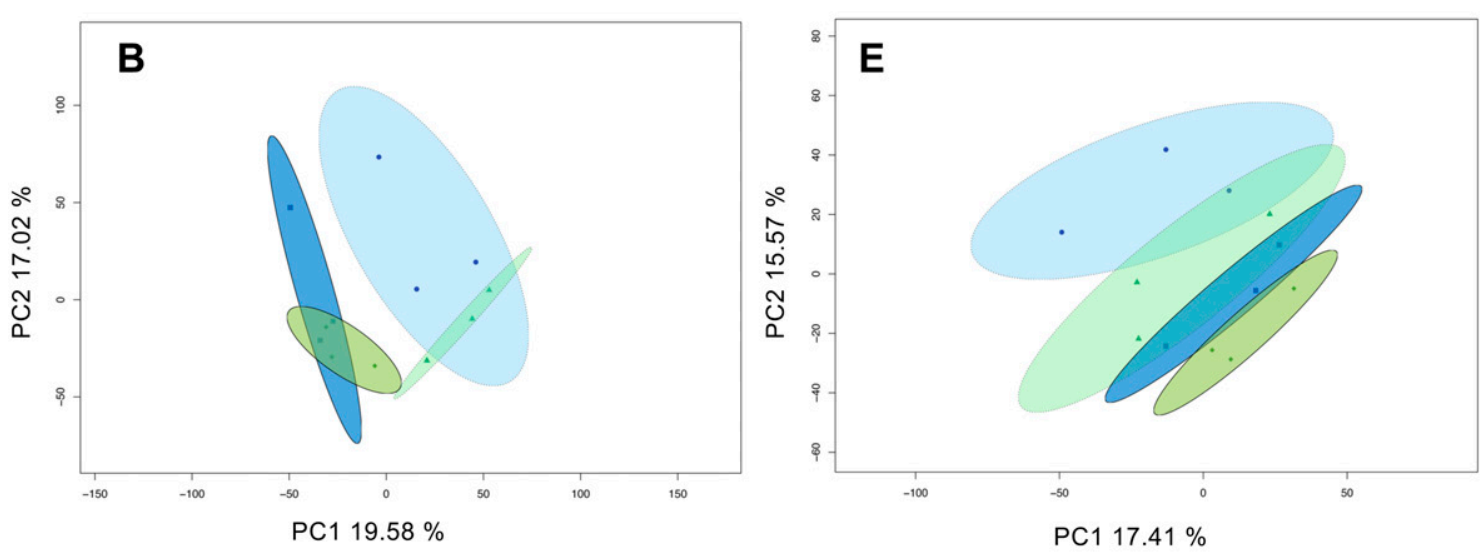

\section{Gaulois}

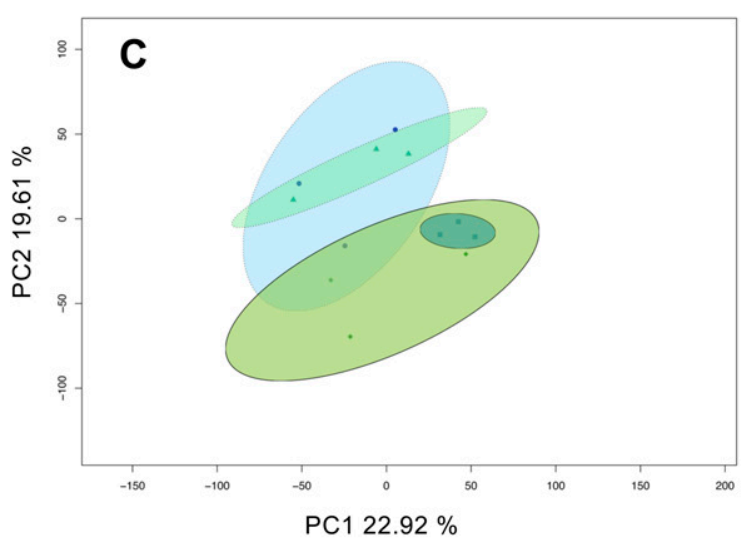

positive ion mode

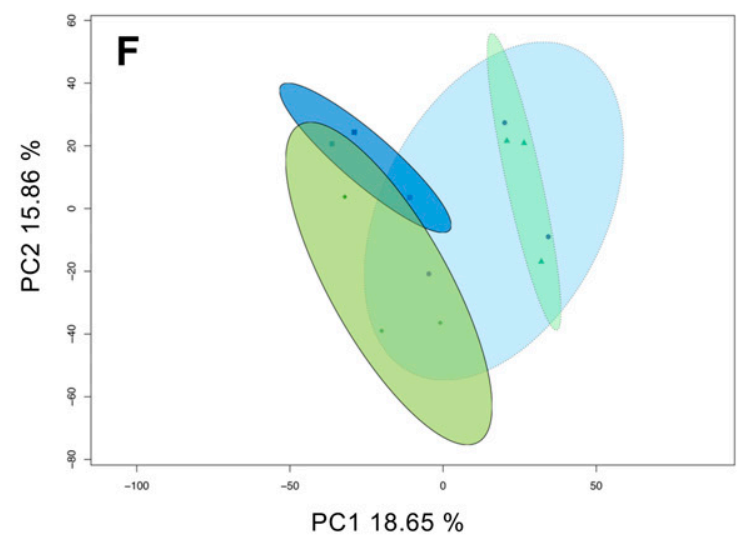

negative ion mode

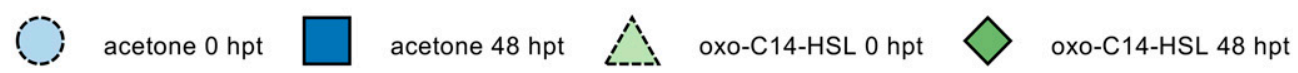

Fig. 5. Metabolome changes upon priming in Golden Promise and Gaulois. A and D, Score plots from principal component analyses (PCA) of metabolite profiles obtained from methanolic extracts of whole in vitro-grown barley plants, pretreated with $6 \mu \mathrm{M} \mathrm{oxo}-\mathrm{C} 14-\mathrm{HSL}$, and treated with chitin (100 $\mu \mathrm{g} / \mathrm{ml})$, in positive and negative ion mode. $\mathbf{B}$ and E, Golden Promise and $\mathbf{C}$ and $\mathbf{F}$, Gaulois show differences in its metabolite profiles between plants pretreated with oxo-C14-HSL (green filled) or acetone control (blue filled) post chitin treatment. Light colors indicate the controls $(0 \mathrm{~h})$, while the darker colors indicate samples harvested $48 \mathrm{~h}$ post treatment (hpt) with chitin. 
well as other differences, e.g., in the signaling pathways, could be the underpinning reasons for the differences in the $A H L$ primability among barley genotypes.

MAPKs are key signaling components that relay extracellular signal perception with intracellular (nuclear) responses (Davis et al. 2000; Widmann et al. 1999). Previously, MAP kinases were associated with priming. Stronger and prolonged activation of AtMPK3 and AtMPK6 was shown to be a key element of priming for enhanced defense response in Arabidopsis (Beckers et al. 2009; Schenk and Schikora 2014; Schikora et al. 2011). Golden Promise primed with oxo-C14-HSL showed stronger and prolonged activity of barley MAPKs after a subsequent treatment with chitin, whereas Gaulois showed similar MAPKs activation after chitin treatment following acetone or oxo-C14-HSL pretreatments (Fig. 4A). This difference in activation patterns could explain, at least partially, the nonresponsiveness of Gaulois to the priming agent and therefore the lack of enhanced papillae and HR occurrence.

Lignification of cell walls plays an important function as constitutive as well as inducible resistance mechanisms against pathogens (Dixon and Paiva 1995; Eynck et al. 2012; Nicholson and Hammerschmidt 1992; Vance et al. 1980). Resistant durum wheat were found to have significant differences in lignin monolignols composition, arabinoxylan substitutions and pectin methylesterification compared with the susceptible ones suggesting that differences in cell wall traits are associated with resistance against Fusarium graminearum (Lionetti et al. 2015). In tomato, resistant varieties have significantly higher content of soluble phenolics and lignin in response to Ralstonia solanacearum than the susceptible varieties. Enhanced accumulation of phenolic compounds and lignin in cell walls after oxo-C14-HSL pretreatment and subsequent pathogen challenge

\section{Golden Promise}

A
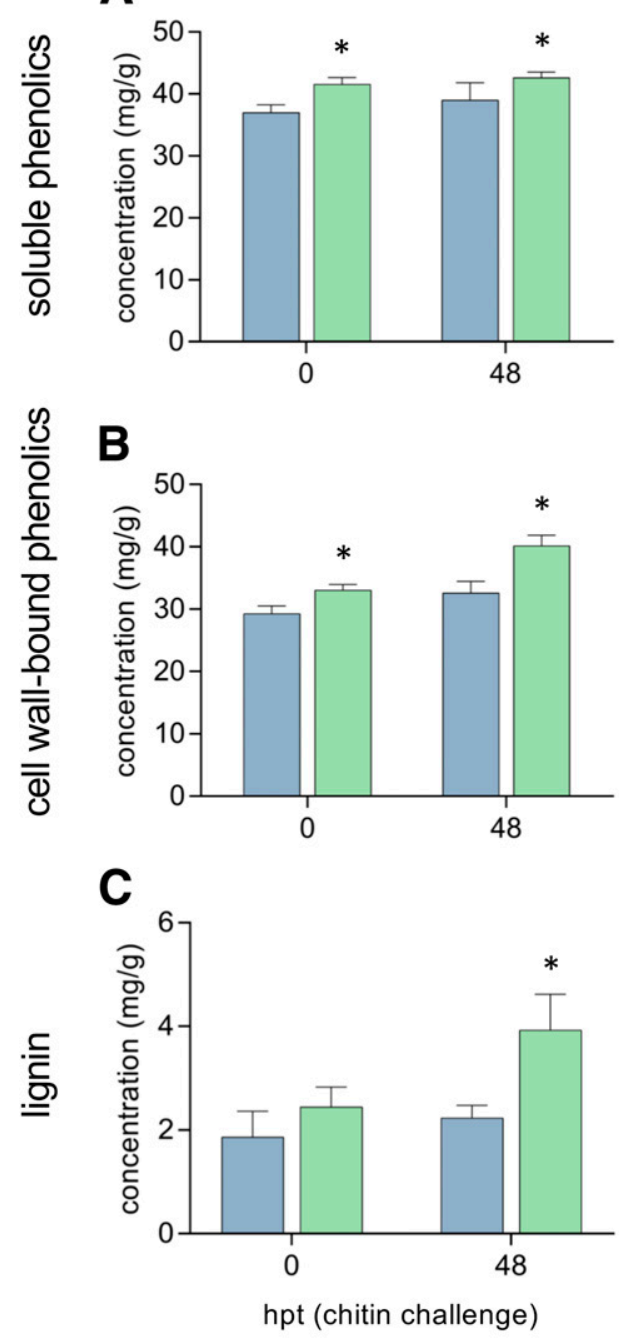

$\square$ acetone

\section{Gaulois}

D

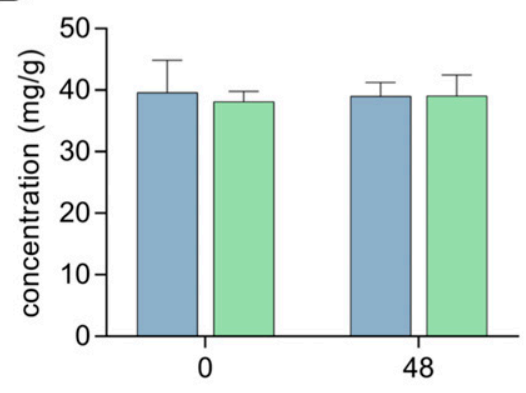

E

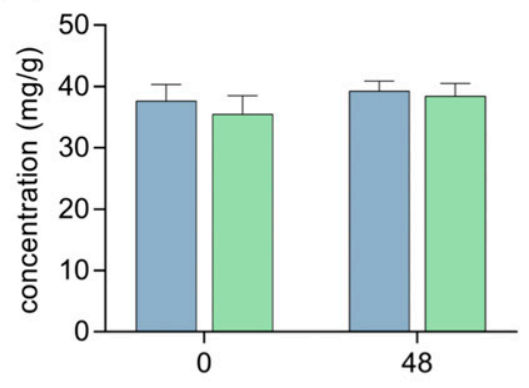

$\mathbf{F}$

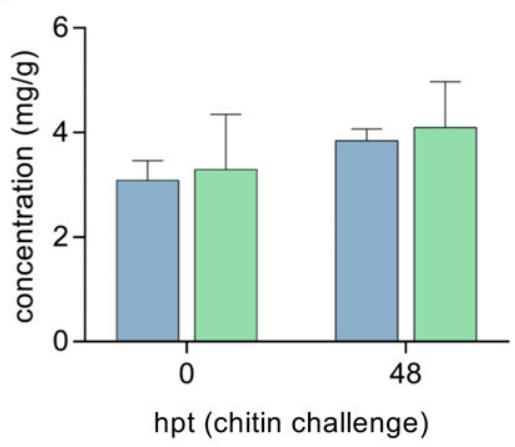

oxo-C14-HSL

Fig. 6. $\mathrm{N}$-acyl homoserine lactone (AHL)-priming has an impact on phenol accumulation in Golden Promise and had no significant impact on Gaulois. Colorimetric quantification of $\mathbf{A}$ and $\mathbf{D}$, soluble phenolic compounds, $\mathbf{B}$ and $\mathbf{E}$, cell wall-bound phenolic compounds, and $\mathbf{C}$ and $\mathbf{F}$, lignin was performed using the Folin-Ciocalteau method with some modifications. Barley seedlings of both genotypes were grown in vitro, pretreated with $6 \mu \mathrm{M}$ oxo-C14-HSL or acetone (solvent control) for 3 days, and subsequently challenged with chitin $(100 \mu \mathrm{g} / \mathrm{ml})$ for $48 \mathrm{~h}$ post treatment (hpt). Both phenolic fractions were extracted from $30 \mathrm{mg}$ of lyophilized plant tissues and normalized to gallic acid. Quantification of lignin was done using alkaline lignin as a standard. The experiment was repeated three times. ${ }^{*}$ indicates $P<0.05$ in Student's $t$ test. Error bars represent standard deviation. 
was proposed as part of the physiological remodeling in primed Arabidopsis plants (Balmer et al. 2015; Schenk et al. 2014). Although, we observed higher accumulation of soluble and cell wallbound phenolics accompanied with enhanced lignification in the Golden Promise genotype (Fig. 6A, B, and C), no difference between the oxo-C14-HSL pretreated and control plants was observed in the Gaulois genotype (Fig. 6D, E, and F). The analysis of the whole metabolome profile was less conclusive. The differences between the Golden Promise and Gaulois genotypes were prominent as were the differences in barley metabolic composition after the challenge with chitin. However, only particular metabolites seem to differ in their abundance between primed and nonprimed plants. We are currently identifying the nature of those compounds.

Whether the inability of the Gaulois genotype to induce AHLpriming response is due to its impaired perception of AHL molecule or impairment in priming response itself remains to be tested. The precise description of the AHL-priming mechanism might be indeed very difficult and require nonbiased approaches such as for example whole transcriptome profiling. Another strategy could be to detect QTL involved in priming through genome wide association studies (GWAS) by screening a larger set of barley accessions as suggested by Wehner et al. (2019).

Conclusions. We showed that the principal mechanism of AHLinduced priming in barley seems similar to the mechanism in Arabidopsis, i.e., it is associated with the activation of MAPKs, enhanced expression of various defense-related genes and remodeling of cell wall structure. Such findings are important, as they open doors to study the mechanisms and the interactions between the plant genetic background and priming. The use of AHL molecules to modulate plant defense clearly needs further optimization and deeper understanding of its mechanisms. Nonetheless, some factors are already known: plant genotype, soil and microbial community compositions, and priming inoculum. Identification of the metabolites that differ between AHL-primable and AHL-nonprimable genotypes should help to increase the efficacy of priming in crop plants and perhaps lead us to new breeding approaches for sustainable crop protection.

\section{LITERATURE CITED}

Ainsworth, E. A., and Gillespie, K. M. 2007. Estimation of total phenolic content and other oxidation substrates in plant tissues using Folin-Ciocalteu reagent. Nat. Protoc. 2:875-877.

Bai, X., Todd, C. D., Desikan, R., Yang, Y., and Hu, X. 2011. N-3-oxo-decanoylL-homoserine-lactone activates auxin-induced adventitious root formation via hydrogen peroxide- and nitric oxide-dependent cyclic GMP signaling in mung bean. Plant Physiol. 158:725-736.

Balmer, A., Pastor, V., Gamir, J., Flors, V., and Mauch-Mani, B. 2015. The 'prime-ome': Towards a holistic approach to priming. Trends Plant Sci. 20: 443-452.

Beckers, G. J., Jaskiewicz, M., Liu, Y., Underwood, W. R., He, S. Y., Zhang, S., and Conrath, U. 2009. Mitogen-activated protein kinases 3 and 6 are required for full priming of stress responses in Arabidopsis thaliana. Plant Cell 21: 944-953.

Bengtsson, T., Ahman, I., Manninen, O., Reitan, L., Christerson, T., Due Jensen, J., Krusell, L., Jahoor, A., and Orabi, J. 2017. A novel QTL for powdery mildew resistance in Nordic spring barley (Hordeum vulgare L. ssp. vulgare) revealed by genome-wide association study. Front. Plant Sci. 8:1954.

Böttcher, C., Krähmer, A., Stürtz, M., Widder, S., and Schulz, H. 2017. Comprehensive metabolite profiling of onion bulbs (Allium cepa) using liquid chromatography coupled with electrospray ionization quadrupole time-offlight mass spectrometry. Metabolomics 13:35.

Böttcher, C., Krahmer, A., Sturtz, M., Widder, S., and Schulz, H. 2018. Effect of cultivar and cultivation year on the metabolite profile of onion bulbs (Allium cepa L.). J. Agric. Food Chem. 66:3229-3238.

Cantalapiedra, C. P., Contreras-Moreira, B., Silvar, C., Perovic, D., Ordon, F., Gracia, M. P., Igartua, E., and Casas, A. M. 2016. A cluster of nucleotide- binding site-leucine-rich repeat genes resides in a barley powdery mildew resistance quantitative trait loci on 7HL. Plant Genome 9:2.

Carisse, K. X., Burnett, P. A., Tewari, J. P., Chen, M. H., Turkington, T. K., and Helm, J. H. 2000. Histopathological study of barley cultivars resistant and susceptible to Rhynchosporium secalis. Phytopathology 90:94-102.

Comadran, J., Kilian, B., Russell, J., Ramsay, L., Stein, N., Ganal, M., Shaw, P., Bayer, M., Thomas, W., Marshall, D., Hedley, P., Tondelli, A., Pecchioni, N., Francia, E., Korzun, V., Walther, A., and Waugh, R. 2012. Natural variation in a homolog of Antirrhinum CENTRORADIALIS contributed to spring growth habit and environmental adaptation in cultivated barley. Nat. Genet. 44:1388-1392.

Conrath, U., Pieterse, C. M. J., and Mauch-Mani, B. 2002. Priming in plant-pathogen interactions. Trends Plant Sci. 7:210-216.

Davis, S., Vanhoutte, P., Pagès, C., Caboche, J., and Laroche, S. 2000. The MAPK/ERK cascade targets both Elk-1 and cAMP response element-binding protein to control long-term potentiation-dependent gene expression in the dentate gyrus in vivo. J. Neurosci. 20:4563-4572.

Dixon, R. A., and Paiva, N. L. 1995. Stress-induced phenylpropanoid metabolism. Plant Cell 7:1085-1097.

Douchkov, D., Lück, S., Johrde, A., Nowara, D., Himmelbach, A., Rajaraman, J., Stein, N., Sharma, R., Kilian, B., and Schweizer, P. 2014. Discovery of genes affecting resistance of barley to adapted and non-adapted powdery mildew fungi. Genome Biol. 15:518.

Eynck, C., Koopmann, B., Karlovsky, P., and Tiedemann, A. 2009. Internal resistance in winter oilseed rape inhibits systemic spread of the vascular pathogen Verticillium longisporum. Phytopathology 99:802-811.

Eynck, C., Seguin-Swartz, G., Clarke, W. E., and Parkin, I. A. 2012. Monolignol biosynthesis is associated with resistance to Sclerotinia sclerotiorum in Camelina sativa. Mol. Plant Pathol. 13:887-899.

Fuqua, W. C., and Winans, S. C. 1994. A LuxR-LuxI type regulatory system activates Agrobacterium Ti plasmid conjugal transfer in the presence of a plant tumor metabolite. J. Bacteriol. 176:2796-2806.

Gantner, S., Schmid, M., Durr, C., Schuhegger, R., Steidle, A., Hutzler, P., Langebartels, C., Eberl, L., Hartmann, A., and Dazzo, F. B. 2006. In situ quantitation of the spatial scale of calling distances and population densityindependent $N$-acylhomoserine lactone-mediated communication by rhizobacteria colonized on plant roots. FEMS Microbiol. Ecol. 56:188-194.

Gower, J. C. 1967. Multivariate analysis and multidimensional geometry. J. Roy. Stat. Soc. D-Sta. 17:13-28.

Heller, J., and Tudzynski, P. 2011. Reactive oxygen species in phytopathogenic fungi: Signaling, development, and disease. Annu. Rev. Phytopathol. 49: 369-390.

Hernandez-Reyes, C., Schenk, S. T., Neumann, C., Kogel, K. H., and Schikora, A. 2014. $\mathrm{N}$-acyl-homoserine lactones-producing bacteria protect plants against plant and human pathogens. Microbiol. Biotechnol. 7:580-588.

Huckelhoven, R., and Kogel, K. H. 2003. Reactive oxygen intermediates in plant-microbe interactions: Who is who in powdery mildew resistance? Planta 216:891-902.

Jaskiewicz, M., Conrath, U., and Peterhansel, C. 2011. Chromatin modification acts as a memory for systemic acquired resistance in the plant stress response. EMBO Rep. 12:50-55.

Jung, H. W., Tschaplinski, T. J., Wang, L., Glazebrook, J., and Greenberg, J. T. 2009. Priming in systemic plant immunity. Science 324:89-91.

Kaplan, H. B., and Greenberg, E. P. 1985. Diffusion of autoinducer is involved in regulation of the Vibrio fischeri luminescence system. J. Bacteriol. 163: 1210-1214.

Kaufman, L., and Rousseeuw, P. J. 2005. Finding Groups in Data: An Introduction to Cluster Analysis vol. 603. Probability and Statistics. John Wiley \& Sons, Hoboken, NJ.

Lionetti, V., Giancaspro, A., Fabri, E., Giove, S. L., Reem, N., Zabotina, O. A., Blanco, A., Gadaleta, A., and Bellincampi, D. 2015. Cell wall traits as potential resources to improve resistance of durum wheat against Fusarium graminearum. BMC Plant Biol. 15:6.

Mathesius, U., Mulders, S., Gao, M., Teplitski, M., Caetano-Anolle's, G., Rolfe, B. G., and Bauer, W. D. 2003. Extensive and specific responses of a eukaryote to bacterial quorum-sensing signals. PNAS 100:1444-1449.

Mauch-Mani, B., Baccelli, I., Luna, E., and Flors, V. 2017. Defense priming: An adaptive part of induced resistance. Annu. Rev. Plant Biol. 68:485-512.

Nicholson, R. L., and Hammerschmidt, R. 1992. Phenolic compounds and their role in disease resistance. Annu. Rev. Phytopathol. 30:369-389.

O'Brien, J. A., Daudi, A., Butt, V. S., and Bolwell, G. P. 2012. Reactive oxygen species and their role in plant defence and cell wall metabolism. Planta 236: 765-779.

Ortiz-Castro, R., Martinez-Trujillo, M., and Lopez-Bucio, J. 2008. N-acylL-homoserine lactones: a class of bacterial quorum-sensing signals alter 
post-embryonic root development in Arabidopsis thaliana. Plant Cell Environ. 31:1497-1509.

Pang, Y., Liu, X., Ma, Y., Chernin, L., Berg, G., and Gao, K. 2009. Induction of systemic resistance, root colonization and biocontrol activities of the rhizospheric strain of Serratia plymuthica are dependent on $\mathrm{N}$-acyl homoserine lactones. Eur. J. Plant Pathol. 124:261-268.

Reif, J. C., Melchinger, A. E., and Frisch, M. 2005. Genetical and mathematical properties of similarity and dissimilarity coefficients applied in plant breeding and seed bank management. Crop Sci. 45:1-7.

Reynolds, A. P., Richards, G., de La Iglesia, B., and Rayward-Smith, V. J. 2006. Clustering rules: A comparison of partitioning and hierarchical clustering algorithms. J Math. Model Algor. 5:475-504.

Romero, C. C. T., Vermeulen, J. P., Vels, A., Himmelbach, A., Mascher, M., and Niks, R. E. 2018. Mapping resistance to powdery mildew in barley reveals a large-effect nonhost resistance QTL. Theor. Appl. Genet. 131:1031-1045.

RStudio Team. 2015. RStudio: Integrated Development for R. RStudio, Boston, MA.

Schenk, S. T., Hernandez-Reyes, C., Samans, B., Stein, E., Neumann, C., Schikora, M., Reichelt, M., Mithofer, A., Becker, A., Kogel, K. H., and Schikora, A. 2014. $\mathrm{N}$-acyl-homoserine lactone primes plants for cell wall reinforcement and induces resistance to bacterial pathogens via the salicylic acid/oxylipin pathway. Plant Cell 26:2708-2723.

Schenk, S. T., and Schikora, A. 2014. AHL-priming functions via oxylipin and salicylic acid. Front. Plant Sci. 5:784.

Schenk, S. T., and Schikora, A. 2015. Lignin extraction and quantification, a tool to monitor defense reaction at the plant cell wall level. Bio. Protoc. 5:e1430.

Schenk, S. T., Stein, E., Kogel, K. H., and Schikora, A. 2012. Arabidopsis growth and defense are modulated by bacterial quorum sensing molecules. Plant Signal. Behav. 7:178-181.

Schikora, A., Schenk, S. T., and Hartmann, A. 2016. Beneficial effects of bacteria-plant communication based on quorum sensing molecules of the $N$-acyl homoserine lactone group. Plant Mol. Biol. 90:605-612.

Schikora, A., Schenk, S. T., Stein, E., Molitor, A., Zuccaro, A., and Kogel, K. H. 2011. $\mathrm{N}$-acyl-homoserine lactone confers resistance toward biotrophic and hemibiotrophic pathogens via altered activation of AtMPK6. Plant Physiol. 157:1407-1418.

Schuhegger, R., Ihring, A., Gantner, S., Bahnweg, G., Knappe, C., Vogg, G., Hutzler, P., Schmid, M., Van Breusegem, F., Eberl, L. E. O., Hartmann, A., and Langebartels, C. 2006. Induction of systemic resistance in tomato by $\mathrm{N}$-acyl-L-homoserine lactone-producing rhizosphere bacteria. Plant Cell Environ. 29:909-918.
Silvar, C., Perovic, D., Nussbaumer, T., Spannagl, M., Usadel, B., Casas, A., Igartua, E., and Ordon, F. 2013. Towards positional isolation of three quantitative trait loci conferring resistance to powdery mildew in two Spanish barley landraces. PLoS One 8:e67336.

Silvar, C., Perovic, D., Scholz, U., Casas, A. M., Igartua, E., and Ordon, F. 2012. Fine mapping and comparative genomics integration of two quantitative trait loci controlling resistance to powdery mildew in a Spanish barley landrace. Theor. Appl. Genet. 124:49-62.

Smith, C. A., Want, E. J., O’Maille, G., Abagyan, R., and Siuzdak, G. 2006. XCMS: Processing mass spectrometry data for metabolite profiling using nonlinear peak alignment, matching, and identification. Anal. Chem. 78: 779-787.

Vance, C. P., Kirk, T. K., and Sherwood, R. T. 1980. Lignification as a mechanism of disease resistance. Annu. Rev. Phytopathol. 18:259-288.

von Rad, U., Klein, I., Dobrev, P. I., Kottova, J., Zazimalova, E., Fekete, A., Hartmann, A., Schmitt-Kopplin, P., and Durner, J. 2008. Response of Arabidopsis thaliana to $N$-hexanoyl-DL-homoserine-lactone, a bacterial quorum sensing molecule produced in the rhizosphere. Planta 229:73-85.

Wehner, G., Kopahnke, D., Richter, K., Kecke, S., Schikora, A., and Ordon, F. 2019. Priming is a suitable strategy to enhance resistance towards leaf rust in barley. Phytobiomes J. 3:46-51.

Wehner, G. G., Balko, C. C., Enders, M. M., Humbeck, K. K., and Ordon, F. F. 2015. Identification of genomic regions involved in tolerance to drought stress and drought stress induced leaf senescence in juvenile barley. BMC Plant Biol. 15:125.

Widmann, C., Gibson, S., Jarpe, M. B., and Johnson, G. L. 1999. Mitogenactivated protein kinase: conservation of a three-kinase module from yeast to human. Physiol. Rev. 79:143-180.

Wright, S. 1968. Evolution and the Genetics of Populations: Genetic and Biometric Foundations Vol. 1. Evolution and the Genetics of Populations: A Treatise. University of Chicago Press, Chicago.

Zarkani, A. A., Stein, E., Rohrich, C. R., Schikora, M., Evguenieva-Hackenberg, E., Degenkolb, T., Vilcinskas, A., Klug, G., Kogel, K. H., and Schikora, A. 2013. Homoserine lactones influence the reaction of plants to rhizobia. Int. J. Mol. Sci. 14:17122-17146.

Zeyen, R. J., Carver, T. L. W., and Lyngkjaer, M. F. 2002. Epidermal cell papillae. Pages 107-125 in: The Powdery Mildews. A Comprehensive Treatise. R. R. Belanger, W. R. Bushnell, A. J. Dik, and T. L. W. Carver, eds. American Phytopathological Society, St. Paul, MN. 